\title{
Random DFB Fiber Laser for Remote (200 km) Sensor Monitoring Using Hybrid WDM/TDM
}

\author{
Daniel Leandro, Veronica deMiguel-Soto, Rosa Ana Perez-Herrera, Mikel Bravo and Manuel Lopez- \\ Amo, Senior Member, IEEE.
}

\begin{abstract}
In this work, a random distributed feedback (DFB) fiber laser is proposed as a multiplexing scheme for ultra-long range measurements (up to $200 \mathrm{~km}$ ). Optical fiber sensors are time and wavelength multiplexed overcoming one of the main limitations of long-range sensing setups, which is their limited multiplexing capability. The direct modulation of the laser's cavity allows the interrogation of sensors by measuring the reflected power for different wavelengths and distances. Fiber Bragg gratings (FBGs) placed at different fiber locations and wavelengths have been interrogated in two different sensor networks. In addition, in order to improve the performance of the system, some features have been analyzed.
\end{abstract}

Index Terms - Random Distributed-feedback fiber laser, Multiplexing, optical fiber sensors, time division multiplexing, wavelength division multiplexing.

\section{INTRODUCTION}

A variety of multiplexing techniques based on different modulation formats have been developed, each one with its own specific advantages for each particular application [1]. These modulation formats generally fall into one of the following categories: Wavelength Division Multiplexing (WDM), Time Division Multiplexing (TDM), Frequency Division Multiplexing (FDM), Coherence Multiplexing (CM) or Polarization Division Multiplexing (PDM) [2]-[4]. Likewise hybrid approaches (simultaneous utilization of two modulation formats inside the same network) have been also considered. The multiplexing capability of WDM schemes can be limited by the available bandwidth, less than $100 \mathrm{~nm}$ [5]. On the other hand TDM schemes have been shown to own simple system structure and high sensitivity [6]. Researches in the recent years have been concentrated on the system expansion by incorporating EDFAs or Raman amplification in the array with hybrid approaches that include more than one multiplexing technique. For example, hybrid TDM/WDM

Manuscript received December, 13, 2015. This work was supported by the Spanish Government project TEC2013-47264-C2-2-R and the FEDER funds.

D. Leandro, V. deMiguel-Soto, R. A. Perez-Herrera, M. Bravo, M. LopezAmo are with Universidad Publica de Navarra, Dept. of Electrical and Electronic Engineering, Campus Arrosadia S/N, E-31006, Pamplona, Spain. (e-mail: daniel.leandro@unavarra.es).

Copyright (c) 2016 IEEE. Personal use of this material is permitted. However, permission to use this material for any other purposes must be obtained from the IEEE by sending a request to pubs-permissions@ieee.org. systems that can multiplex hundreds of sensors in a single network have been validated theoretically. Simulations show that up to 1000 weak-reflection fiber Bragg gratings can be inserted in a single fiber using optical wavelength timedomain reflection (OWTDR) technology [7]. Large-scale interferometric sensor multiplexing schemes have been also published recently, theoretically proving the inclusion of 4096 sensors in an amplified network [8]. Other approach based on chaotic laser sources provides fault detection with a spatial resolution of $2.8 \mathrm{~cm}$ at the cost of lower multiplexing capability [9].

In addition, Optical Time Domain Reflectometry (OTDR) has been demonstrated to achieve fully distributed sensing systems which are be able to find the positions of any backscattered/reflected event along the length of a fiber. These systems are based on an optical pulse which is launched into the fiber under test. A high sensitivity photodetector detects the backscattered light intensity produced by the pulse along the fiber. Light backscattering/reflections occur due to optical fiber discontinuities and also because of small variations of the refractive index of the fiber known as Rayleigh backscattering [10]. Taking advantage of this technology, another simple but effective hybrid approach uses an OTDR to achieve TDM in combination with a tunable filter used for the wavelength selection [11]. OTDR techniques have been also used in ultralong remote sensor systems demonstrating a sensing distance limit of $253 \mathrm{~km}$ for a single sensor [12]. It must be emphasized that the tradeoff between the pulse width and sensing distance is one of the main drawbacks of this technique in order to interrogate long distances. Up to date, few sensing proposals have surpassed $150 \mathrm{~km}$ of sensing range [13] but the majority of them present limited multiplexing capability in terms of position, number and type of sensors (most can interrogate just FBGs at a single location). In this manner, many works have focused on maximizing the number of sensors multiplexed and others have looked for interrogating a sensor located as far as possible. However, in this work, the particular properties of random distributed feedback (DFB) lasers have been explored in the design of a new ultra long-range sensor system, with higher multiplexing capability than others long range setups (in terms of type of sensors and its location along the cavity). 
Random DFB fiber lasers have been subject of intense theoretical and experimental investigations [14] during the last years due to their potential differences when compared to conventional lasers. In this work, its mode-less behavior [15], high power and stability [16], together with the capability of being internally modulated without frequency restrictions [17] are used in the design of a scheme for quasi-distributed fiber optic sensors multiplexing.

Exploiting these singular properties, a new random DFB laser has been designed to achieve ultra-long range sensor interrogation in a multiplexing scheme. Several FBGs have been remotely monitored up to $200 \mathrm{~km}$ away from the monitoring station with approximately $44 \mathrm{~m}$ of spatial resolution. Preliminary results presented in [18] have been extended by modifying the filtering process and studying the influence of different parameters such as the laser bandwidth, modulation values, etc. Results show that random DFB fiber lasers can have a great potential for remote sensing applications when combined with OTDR techniques.

\section{EXPERIMENTAL SETUP AND PRINCIPLE OF OPERATION}

\section{A. Laser design}

The experimental setup of the sensor multiplexing system can be divided in two different parts: the fiber laser itself and the sensor network to be monitored. A random distributed feedback fiber laser design is employed due to its particular properties such as good stability, high power inside the cavity and outstanding performance when modulated. Additionally, the half-open cavity architecture allows the laser cavity to be used as part of the sensor network. Random DFB fiber lasers are based on the distributed amplification of the Rayleigh backscattering along the fiber. In this context, random DFB schemes typically consists of two single mode fibers (SMF) branches which act as distributed mirrors [14]. In the case of using Raman amplification, the SMF of the cavity also acts as the active medium for the amplification due to the Raman scattering effect. Similar performance can be achieved using a single-arm configuration, obtained by replacing one of the fiber arms by a reflector [16]. In this work, the single-arm configuration is used. Thus, the fiber cavity also serves as the sensing network in which numerous sensors can be monitored.

Moreover, the reflector used in the setup is designed to modulate and select the wavelength of operation of the laser. It consists of a fiber loop formed by an optical circulator. Two different techniques have been used to select the wavelength of operation of the laser. In the first experiment, a tunable FBG is connected using a four-port circulator as depicted in Fig. 1(a) with the aim of filtering and consequently selecting the wavelength of the laser. Another approach is used in the second experiment with the aim of automating the measurement process and increasing the wavelength range of the laser. In this case, a programmable filter is placed at the port 3 of the circulator as can be seen in Fig. 1(b). An optical coupler is used to extract a $30 \%$ of the power circulating inside the loop to be detected and analyzed in an oscilloscope. The remaining $70 \%$ of the light is forwarded into the loop to be modulated by an electro-optical amplitude modulator. A signal generator is connected to the modulator creating a pulse train with a pulse repetition rate of $450 \mathrm{~Hz}$ and a pulse duration of $0.83 \mu \mathrm{s}$ and $0.44 \mu \mathrm{s}$ for the first and second experiment respectively. Finally, the light passes through the circulator into the laser's cavity where it is combined with the $1445 \mathrm{~nm}$ pump laser. The single-mode fiber cavity consists of 170 and $200 \mathrm{~km}$ of SMF (for the first and second experiment respectively) as shown in Fig. 1.

As a result, a tunable and amplitude-modulated random DFB fiber laser emission is obtained.
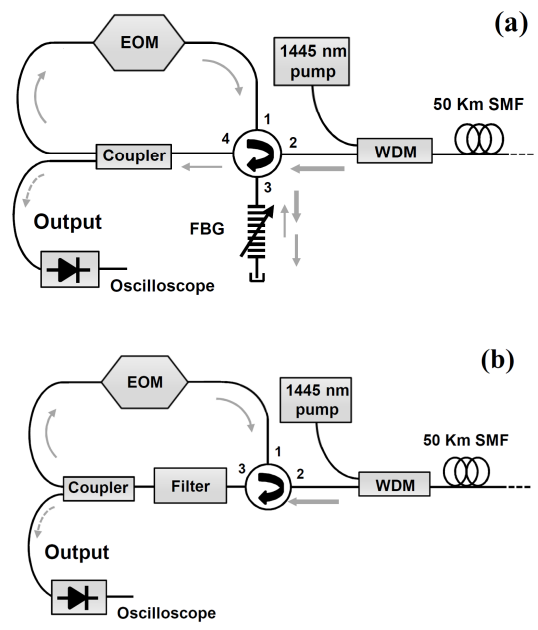

Fig. 1. Experimental setup of the proposed fiber laser in the (a) first and (b) second experiment. EOM: Electro-optical modulator, WDM: Wavelength division multiplexer, SMF: Single mode fiber, FBG: Fiber Bragg grating.

\section{B. Sensor network configuration}

The second part in which the sensing system can be divided is the sensor network itself. As previously stated, the single mode fiber of the laser's cavity is also used as a channel where different fiber optic sensors can be multiplexed. The proposed system can interrogate optical sensors operating in reflection and monitor them using time and wavelength-division multiplexing. Therefore, to validate the system as long-range sensor multiplexing scheme, the sensor network will include fiber Bragg gratings located at different wavelengths and distances from the monitoring station.

The design of the network can include sensors at any position of the cavity up to $200 \mathrm{~km}$. Optical couplers are inserted in the fiber channel to extract a percentage of the light to interrogate sets of sensors. An exception is the last group of sensors, which are directly placed at the end of the cavity. It is important to note that the coupling ratio $k$ of the optical couplers selected is an essential aspect in the performance of the setup. Higher $k$ values will increase the power extracted from the main cavity and the reflected signal; but the system's maximum range will decrease. It must be also taken into consideration that a random DFB fiber laser requires a distributed reflector (SMF fiber in our case) to operate. In this work, the system detects the peak reflections given by the sensors along the cavity. Consequently, including a strong 
reflection in the cavity will change the laser's behavior from a Random DFB to a standard Raman fiber laser with a FabryPérot cavity. This situation must be avoided by limiting the strongest reflection (choosing the appropriate $k$ for each optical coupler) to a value in which the laser remains stable in the random regime. In addition, every fiber end was immersed in refractive index matching gel to avoid undesired reflections.

In this work, two different experiments have been carried out to validate the system as sensor multiplexing scheme. In accordance, two sensor networks have been designed. The first one, depicted in Fig. 2(a), consists of four sets of sensors located at the kilometers 50,100, 150 and 170. The coupling ratios used in the first experiment are $1 \%, 5 \%$ and $30 \%$ for the optical couplers located at the kilometer 50, 100 and 150 as displayed in Fig. 2 (a). In order to demonstrate the TDM feature, the sensors are composed by four pairs of FBGs with the same Bragg wavelengths: $\lambda_{1}=1545.6 \mathrm{~nm}, \lambda_{2}=1550 \mathrm{~nm}$, $\lambda 3=1547 \mathrm{~nm}$ and $\lambda_{4}=1548.6 \mathrm{~nm}$. It is also placed a single FBG which has a Bragg wavelength of $\lambda_{5}=1545.1 \mathrm{~nm}$. The reflectivity of the FBGs is higher than $90 \%$ and the full width at half maximum (FWHM) is $0.2 \mathrm{~nm}$ except for the tunable FBG used in $\lambda_{4}$ that is $0.3 \mathrm{~nm}$.

In the second experiment, the main purpose is to use a wider wavelength range and to monitor the sensors at longer distances. In accordance, the coupling ratios chosen for the optical couplers located at the kilometers 50,100 and 150 are $1 \%, 5 \%$ and $10 \%$ respectively as depicted in Fig. 2 (b). In this case, the FBGs used have the following Bragg wavelengths: $\lambda_{1}=1545.6 \mathrm{~nm}, \lambda_{2}=1550 \mathrm{~nm}, \lambda_{3}=1547 \mathrm{~nm}, \lambda_{4}=1548.6 \mathrm{~nm}$, $\lambda_{5}=1542.4 \mathrm{~nm}$. The Bragg wavelengths of the two FBGs located at the end of the channel illustrated in Fig. 2 (b) are $\lambda_{6}=1541.2 \mathrm{~nm}$ and $\lambda_{7}=1543.6 \mathrm{~nm}$.

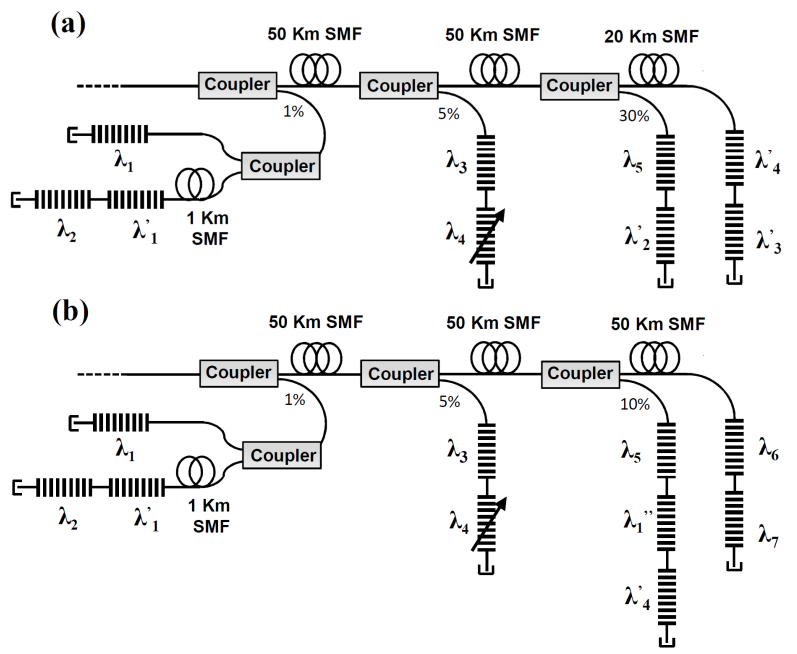

Fig. 2. Experimental setup of the sensor network to be monitored in the (a) first and (b) second experiment.

\section{Principle of operation}

The principle of operation of the multiplexing system relies on the particular properties of random DFB lasers such as its outstanding performance when modulated internally [17]. Additionally, high peak power values are achieved inside the cavity [19], reaching the laser's maximum power around the $40^{\text {th }}$ kilometer in our scheme. High power and wavelength stabilities are also attained due to the absence of longitudinal modes [14]. The laser is wavelength-tuned by the filter placed in the loop mirror and the EOM modulates the light before reentering into the cavity again. In the same manner as an optical time-domain reflectometer, due to the pulse modulation, the reflected power along the fiber can be measured using an oscilloscope. Therefore, a voltage vs time (i.e. distance) profile is obtained for the wavelength set. This measurement can be repeated for different wavelengths by tuning the laser's wavelength, getting the reflected power along the fiber for a wavelength range. In this manner, reflections can be detected in the cavity at different wavelengths. Consequently, if a FBG is placed in the cavity, a reflection peak will arise when the laser's wavelength matches the FBG wavelength. Since the reflected wavelength of the FBGs vary with physical parameters (e.g. temperature or strain), the wavelength of the reflections will encode the information of the sensors. Therefore, fiber optic sensors can be interrogated at any position in the cavity (if they operate in reflection). That implies that sensors can be wavelength and time-multiplexed since for identical wavelength, sensors can be identified by their position along the fiber. Likewise, FBG sensors placed at a close location (given by the pulse duration) can be wavelength-multiplexed. The main factor to be taken into account for an adequate performance of the system is, as previously mentioned, the control of the reflection peaks to keep the laser operation in the random regime.

As a proof-of-concept of the proposed system, two sensor networks have been remotely interrogated for different sensor configurations and distances (170 and $200 \mathrm{~km})$.

\section{EXPERIMENTAL RESULTS}

\section{A. First experiment}

In order to validate the system as ultra-long range remote sensor multiplexing scheme, two different experiments have been carried out. The first one uses a tunable FBG as filtering element (Fig. 1(a)) to interrogate the sensor network depicted in Fig. 2(a). In this case, the FWHM of the filter is $0.2 \mathrm{~nm}$ and the wavelength range for the laser is limited between 1545$1550 \mathrm{~nm}$. The pulse-width of the modulation is $0.83 \mu \mathrm{s}$.

Before carrying out the experiment, the operation under random regime of the laser was confirmed for the whole wavelength range. Fig 3 illustrates the optical spectrum of the emission line measured at an optical spectrum analyzer (OSA) when the tunable FBG was set to $1545 \mathrm{~nm}$ for a pump power of $1.4 \mathrm{~W}$. To validate the random operation of the laser, the self-beating of the line was detected by a photo-detector and measured with an electrical spectrum analyzer (ESA). The absence of longitudinal modes in Fig. 4 indicates that the laser keeps operating in random regime even if the laser's wavelength matches the Bragg wavelength of the sensors. That indicates that the behavior of the laser is not noticeably affected by the FBGs of the sensor network. 


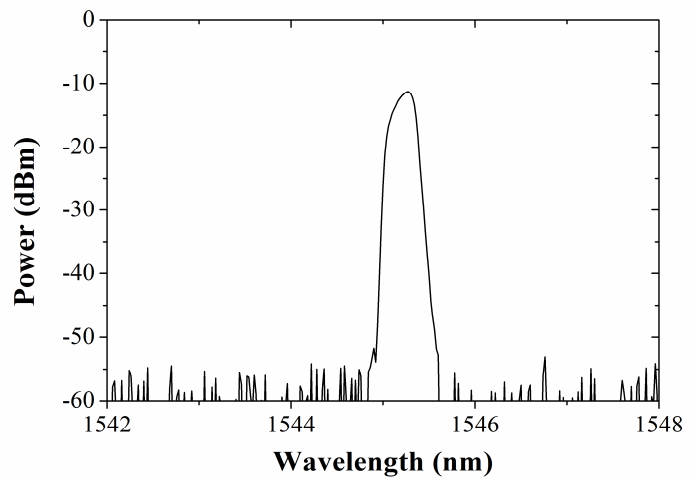

Fig. 3. Optical spectrum of the random DFB fiber laser (resolution $=0.01$ $\mathrm{nm})$.

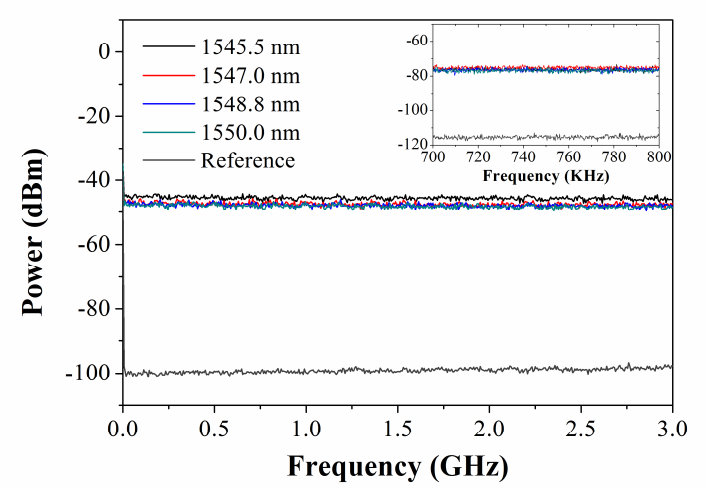

Fig. 4. Self-beating signal of the emission line for different wavelengths. ESA resolution $=300 \mathrm{kHz}(300 \mathrm{~Hz}$ Inset).

After confirming the adequate performance of the laser for the wavelength range under study $(1545-1550 \mathrm{~nm})$, the measurements were taken with a spatial resolution of $85 \mathrm{~m}$ and a wavelength step of $0.05 \mathrm{~nm}$ (longer steps were used at the sensor-free zones to shorten the measurement process). The results obtained are presented in Fig. 5(a) and (b). It can be clearly seen that every sensor can be unambiguously identified by their position and wavelength in the network. In this manner, FBGs located at the same wavelength can be identified by its position along the fiber and sensors located at the same point can be wavelength-multiplexed. The inset in Fig. 5(b) shows a zoom in which the two sensors located $1 \mathrm{~km}$ away at $1545.6 \mathrm{~nm}$ can be easily identified.

A simple validation of the appropriate operation of the system under sensor variations has been also carried out. An axial strain of $1000 \mu \varepsilon$ was applied to the sensor located at $\lambda_{3}$ '. Figure 6 shows the wavelength variation of $\operatorname{FBG}\left(\lambda_{3}\right)$ and $\operatorname{FBG}\left(\lambda_{3}{ }^{\prime}\right)$ located at kilometer 100 and 170 respectively when the strain was applied to $\operatorname{FBG}\left(\lambda_{3}{ }^{\prime}\right)$. It is evident that the wavelength shift of $\operatorname{FBG}\left(\lambda_{3}{ }^{\prime}\right)$ behaves correctly and it does not affect the behavior of $\operatorname{FBG}\left(\lambda_{3}\right)$. Therefore, as a proof of concept, the system has been validated as sensor-multiplexing network with a spatial resolution of $85 \mathrm{~m}$ and a maximum distance of $170 \mathrm{~km}$.
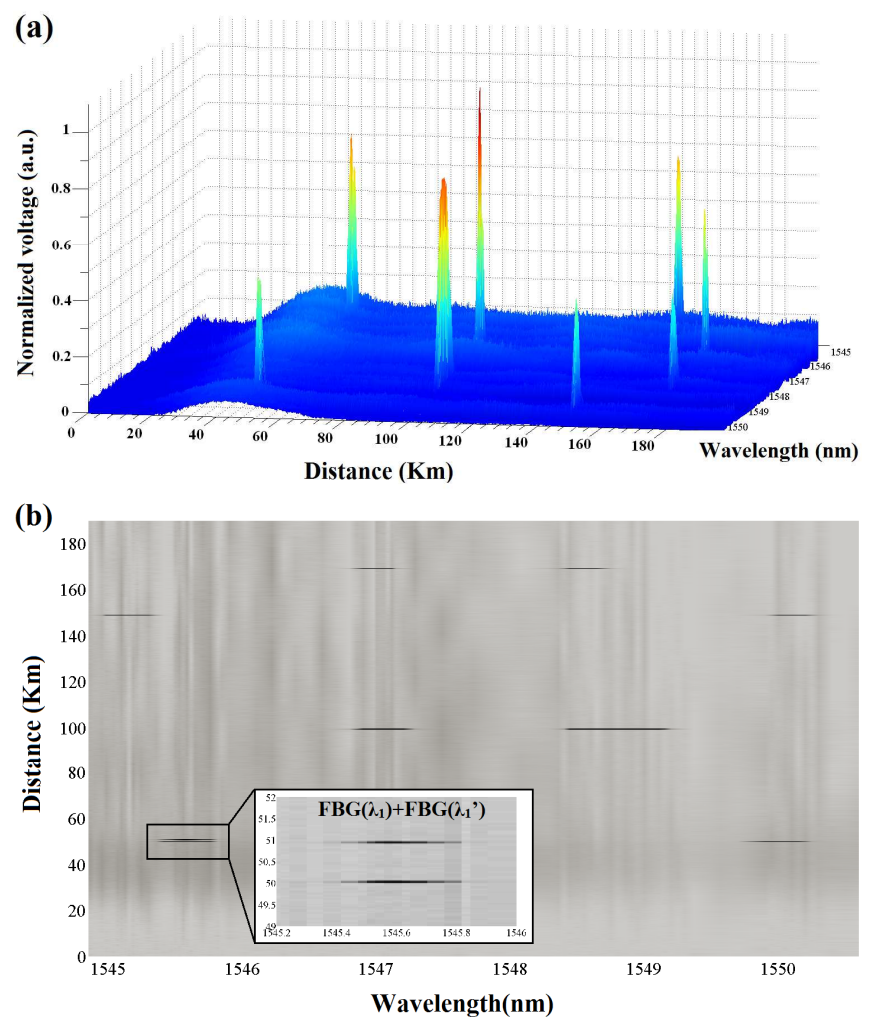

Fig. 5. Experimental results obtained for the $170 \mathrm{Km}$-setup.
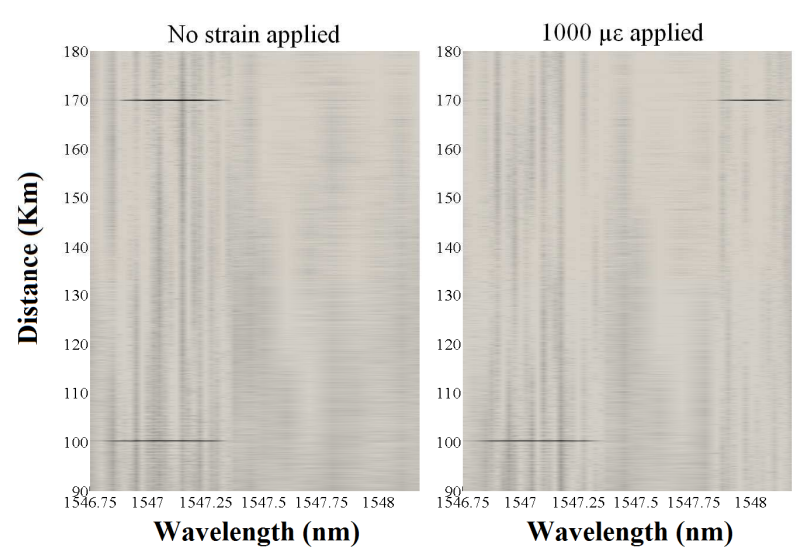

Fig. 6. $\operatorname{FBG}\left(\lambda_{3}\right)$ and $\operatorname{FBG}\left(\lambda_{3}{ }^{\prime}\right)$ wavelength response when $1000 \mu \varepsilon$ are applied to $\operatorname{FBG}\left(\lambda_{3}{ }^{\prime}\right)$.

\section{B. Second experiment}

In order to improve the multiplexing capability of the system by increasing the spatial resolution, the wavelength range and the maximum range of the network, a second experiment was carried out. In this case a programmable filter was used as the filtering element instead of a tunable FBG for the selection of the laser's wavelength. Initially, a study of the bandwidth of the filtering process with the aim of find the value in which the reflection of the sensors is higher was carried out. Figure 7 displays the results, showing an increment of the reflected signal with the bandwidth increment. In accordance, a FWHM of $0.38 \mathrm{~nm}$ was chosen for the filter, obtaining the optical spectrum displayed in Fig. 8. 


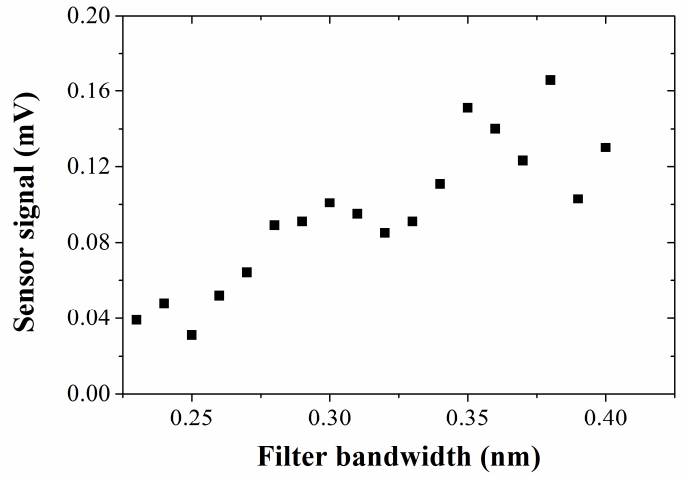

Fig. 7. Reflection given by a FBG for different filter bandwidth values.

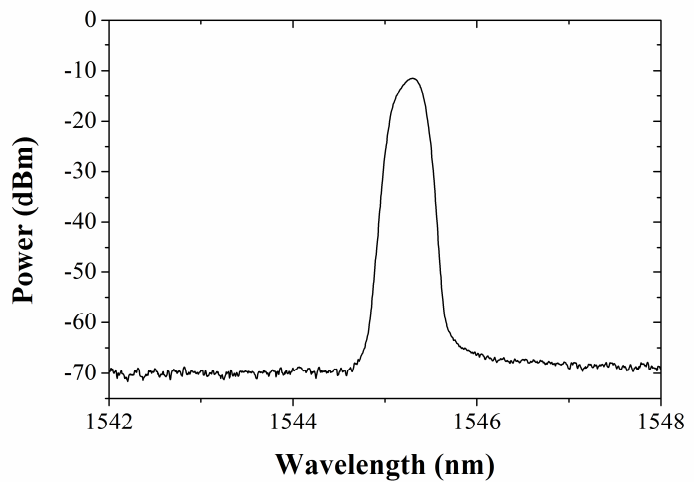

Fig. 8. Optical spectrum of the laser configuration used in the second experiment.

On the other hand, the pulse width has to be narrowed to improve the spatial resolution. In order to check that the system behavior is not affected by the narrowing of the pulse width, the results of the reflection detected for a FBG located at the $\mathrm{km} 50$ for different pulse widths are presented in Fig. 9. It can be seen that the reflected amplitude is constant except for the last case. That drop, together with the pulse deformation (the pulse shape is rectangular) is due to the bandwidth limit of the oscilloscope. In any case, for the system configuration of the first experiment, the spatial resolution can be straightforwardly improved from 85 to $45 \mathrm{~m}$ having a slight decrease in the amplitude. Consequently, the pulse width set for this experiment was $0.44 \mu \mathrm{s}$.

The next factor to be improved was the wavelength range. It has to be taken into account that there is a strong dependence of the Raman gain with wavelength as depicted in Fig. 10 (triangular samples). As a result, for example, if the Raman pump is configured to measure reflections at $1544 \mathrm{~nm}$ (maximum gain), it is possible that a reflection located at a wavelength with smaller gain value is not detected. In the same manner, if the system is properly set for measuring sensors at wavelengths with lower gain, it might occur that the reflections located at $1545 \mathrm{~nm}$ are strong enough to destabilize the laser (leaving the random regime). To avoid these effects, the automated filter was programmed to apply an attenuation value that keeps the laser operating at a constant power. The results of the system with and without the attenuation control can be seen in Fig. 10 (dots and square samples). As a consequence, the pump power required in this experiment was higher, being needed $1.60 \mathrm{~W}$. This power equalization allowed the wavelength increase of the network to $1540-1550 \mathrm{~nm}$.

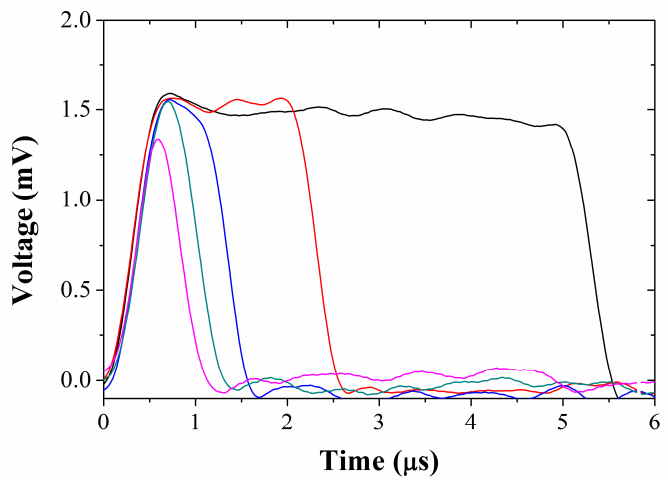

Fig. 9. Reflection given by a FBG for different pulse widths.

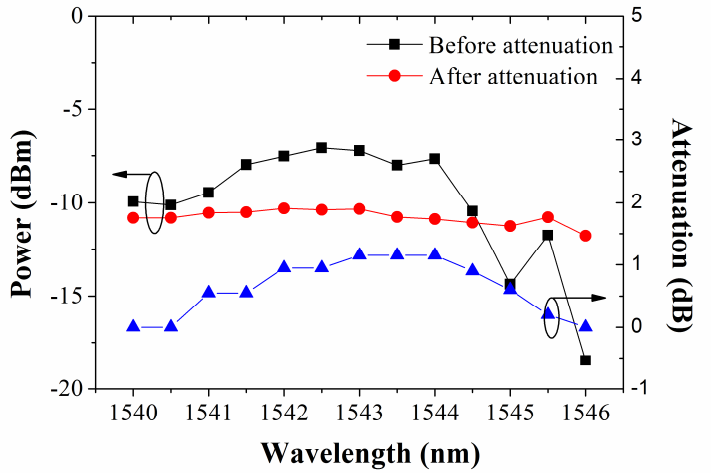

Fig. 10. Attenuation profile and optical power detected before and after the application of the attenuation control.
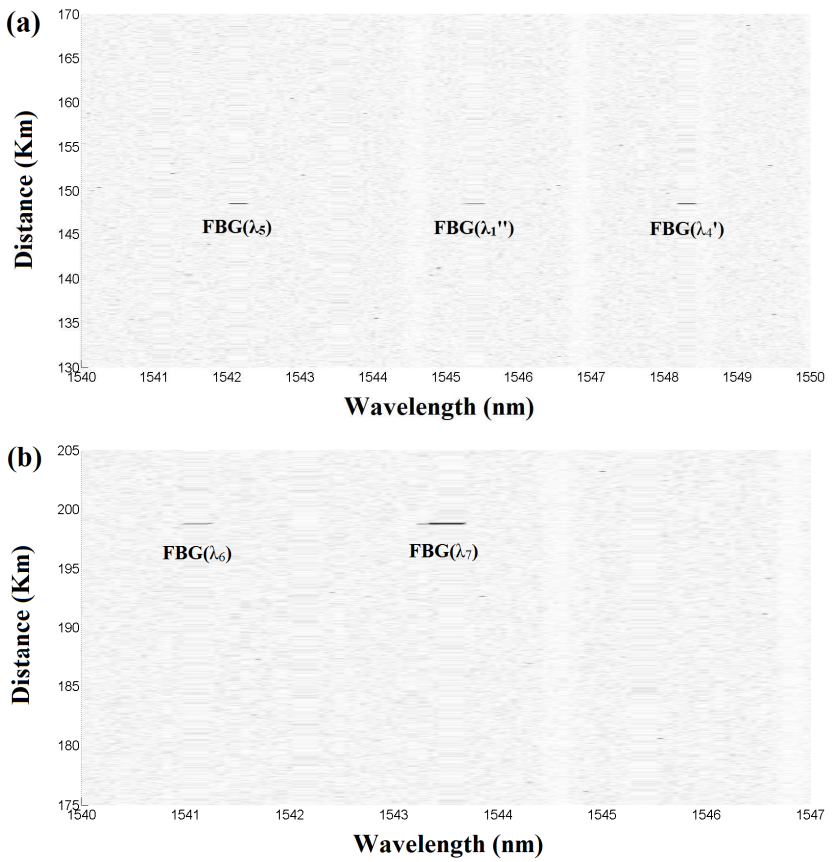

Fig. 11. Experimental results for the FBGs located at the kilometers (a) 150 and (b) 200 . 
Finally, the network was measured for a wavelength range between $1540-1550 \mathrm{~nm}$, a spatial resolution of $45 \mathrm{~m}$ and a maximum distance of $200 \mathrm{~km}$. The results of the FBGs located at the $\mathrm{km} 200$ and 150 can be seen in Fig. 11(a) and (b). The sensors can be identified even after improving the spatial resolution from 85 to $45 \mathrm{~m}$, doubling the wavelength range and increasing the sensing distance to $200 \mathrm{~km}$.

In order to evaluate the repeatability of the measurements, the results obtained from a FBG sensor located at $1547 \mathrm{~nm}$ for nine consecutive sweeps are presented in Fig. 12. As expected, the peak of the trace varies between two adjacent samples, showing a good repeatability.

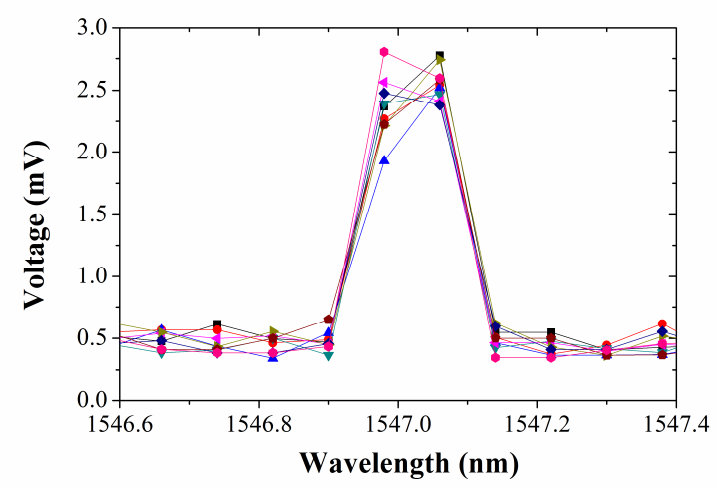

Fig. 12. Results obtained from nine consecutive sweeps to evaluate the repeatability of the measurements.

Referring to the relationship between the number of sensors that can be multiplexed using this technique and the performance of the system, there are several aspects that must be taken into account. Initially, it is worth mentioning that the spatial resolution is not affected by the number of sensors of the system. Depending on the physical parameters to be measured and the type of sensors, a different amount of FBGs can be included in each branch. The inclusion of sensors in the already set branches would not affect the performance of the system. However, if more optical couplers are placed in the main cavity to insert more sensors, the maximum distance of the system will decrease. In this manner, the maximum sensing distance depends on the amount of power extracted from the cavity. It should be mentioned that there is no limitation on the location of the sensors along the cavity. In the proposed setups the couplers have been placed at the splicing points between reels for simplicity, but the sensors can be located at any position. However, including an optical coupler will have different impact in the system depending on its location along the cavity. For example, if arrays of sensors are included before the kilometer 50 (which is the point where most part of the Raman amplification is depleted) the pump power will be critically affected. This would have an important repercussion in the amplification process, significantly reducing the maximum range of the system.

\section{CONCLUSIONS}

In this study, for the first time, a random DFB fiber laser has been used to create and validate a new sensor system for time and wavelength division multiplexing. To achieve this, the particular properties of Random lasers have been exploited, like its good performance when modulated or high stability. The modulation of the laser's cavity allows the sensors to be identified by means of time-domain reflectometry. Moreover, the reflected signal vs distance profile has been measured at different wavelengths by tuning the emission wavelength of the laser. Consequently, this work overcomes one of the main drawbacks of ultra-long range remote sensing systems, that is their very limited multiplexing capability. Using this setup, sensors located at the same wavelength can be identified by their position in the network. In the same manner, sensors closely spaced can be wavelength-multiplexed.

Two different experiments were carried out. Initially, a simple proof of concept was performed, using a tunable FBG as the filtering element of the laser. As a result, 9 FBGs (located at wavelengths between 1545-1550 nm) were monitored up to $170 \mathrm{~km}$ away from the monitoring station with a spatial resolution of $85 \mathrm{~m}$. In the second experiment, the filtering device was replaced by a programmable filter. Additionally, a study of the different properties of the system allowed several improvements over the first experiment. In this manner, 10 FBG sensors were monitored $200 \mathrm{~km}$ away with a spatial resolution of $45 \mathrm{~m}$ and a wavelength range between $1540-1550 \mathrm{~nm}$. It is worth saying that the number of sensors measured was limited by the material availability but the system allows a higher number of FBG to be directly measured. However, due to the complex laser dynamics, further work is required to reliably estimate the maximum number of sensor that can be multiplexed.

More advanced reflectometry techniques and equipment could straightforwardly improve the performance of the system. For example, a wider optical spectrum can be measured by an appropriate control of the gain profile. In conclusion, a new sensing application for random DFB fiber lasers is proposed by exploiting its singular properties. Preliminary results show a greater multiplexing ability of the scheme compared to other ultra-long sensing setups. Additionally, authors consider that further work can significantly improve the capabilities of the system.

\section{REFERENCES}

[1] M. López-Amo, J.M. López-Higuera; "Multiplexing Techniques for FBG Sensors," in Fiber Bragg Grating Sensors: Research Advancements, Industrial Applications and Market Exploitation; 1st ed. Ed. Bentham Science Publishers Ltd, (2011).

[2] R.A. Perez-Herrera, M. Lopez-Amo, "Fiber optic sensor networks," Opt. Fiber Technol., vol. 19, no. 6, pp. 689-699, Dec. 2013.

[3] G.A. Cranch, P.J. Nash, "Large-scale multiplexing of interferometric fiber-optic sensors using TDM and DWDM," J. Lightw. Technol., vol. 19, no. 5, pp. 687-699, May. 2001.

[4] G. Fang, T. Xu, F. Li, "Heterodyne interrogation system for TDM interferometric fiber optic sensors array," Opt. Commun., vol. 341, pp. 74-78, Apr. 2015.

[5] H. Chenyuan, W. Hongqiao, B. Wei, "A Novel Interrogation System for Large Scale Sensing Network With Identical Ultra-Weak Fiber Bragg Gratings," J. Lightw. Technol., vol. 32, no. 7, pp. 1406-1411, Apr. 2014. 
[6] C.K. Kirkendall, A. Dandridge, "Overview of high performance fiberoptic sensing," J Phys. D Appl. Phys., vol. 37, no. 18, pp. R197-R216, Sept. 2004.

[7] M. Zhang, Q. Sun, Zi W., X.Li, H. Liu and D. Liu, "A large capacity sensing network with identical weak fiber Bragg gratings multiplexing," Opt. Comm., vol. 285, no. 13-14, pp. 3082-3087, Jun. 2012.

[8] Y. Liao, E: Austin, P.J. Nash, S.A. Kingsley, D.J. Richardson, "Highly Scalable Amplified Hybrid TDM/DWDM Array Architecture for Interferometric Fiber-Optic Sensor Systems," J. Lightw.Technol., vol.31, no.6, pp.882-888, Mar., 2013.

[9] Y. Luo, L. Xia, W. Li, Z. Xu, Q. Sun, and D. Liu, "Optical Chaos and Hybrid WDM/TDM Based Large Capacity Quasi-distributed Sensing Network with Real-time Fiber Fault Monitoring," Opt. Express, vol. 23, no. 3, pp. 2416-2423, Jan. 2015.

[10] N. Niharika, M. Basu, S.K. Ghorai, "Detection and monitoring of multiple cracks using distributed fiber optic sensor" in Proc. SPIE ICACCCT 7019204, 2014, pp. 809-812.

[11] L.C.G. Valente,.M.B. Braga, A.S. Ribeiro, R.D. Regazzi, W. Ecke, C. Chojetzki, R. Willsch, "Combined time and wavelength multiplexing technique of optical fiber grating sensor arrays using commercial OTDR equipment," IEEE Sens. J. , vol.3, no.1, pp.31-35, Feb. 2003.

[12] M. Bravo, J. M. Baptista, J.L. Santos, M. Lopez-Amo, et al, "Ultralong $250 \mathrm{~km}$ remote sensor system based on a fiber loop mirror interrogated by an optical time-domain reflectometer," Opt. Lett., vol. 36, no. 20, pp 4059-4061, Oct. 2011.

[13] M. Fernandez-Vallejo, M. Lopez-Amo, "Optical fiber networks for remote fiber optic sensors," Sensors, vol. 12, no. 4, pp. 3929-3951, Mar. 2012.

[14] S.K. Turitsyn, S.A. Babin, A.E. El-Taher, P. Harper, D.V. Churkin, S.I. Kablukov, J.D. Ania-Castañon, et al., "Random distributed feedback fibre laser," Nat. Photonics, vol. 4, no. 4, pp. 231-235, Feb. 2010.

[15] M. Fernandez-Vallejo, S. Rota-Rodrigo, M. Lopez-Amo, "Comparative study of ring and random cavities for fiber lasers," Appl. Optics, vol. 53, no. 16, pp. 3501-3507, Jun. 2014.

[16] S.K. Turitsyn, S.A. Babin, D.V. Churkin, I.D. Vatnik, M. Nikulin, E.V. Podivilov, "Random distributed feedback fibre lasers," Phys. Reports, vol. 542, no. 2, pp. 133-193, Sep. 2014

[17] M. Bravo, M. Fernandez-Vallejo, M. Lopez-Amo, "Internal modulation of a random fiber laser," Opt. Lett., vol. 38, no. 9, pp. 1542-1544, May. 2013.

[18] D. Leandro, R. Perez-Herrera, M. Bravo, et al., "Time and wavelength division multiplexing scheme for ultra-long sensing based on a cavitymodulated random DFB fiber laser," in Procc of SPIE, 2015. pp. 9634 963415.

[19] D.V. Churkin, A.E. El-Taher, I.D. Vatnik, et al., "Experimental and theoretical study of longitudinal power distribution in a random DFB fiber laser," Opt. Express, vol. 20, no. 10, pp. 11178-11188, May. 2012.

Daniel Leandro was born in Lumbier, Spain, in November 1984. He received the telecommunication engineering degree and communication master degree from the Universidad Publica de Navarra, Spain in 2010 and 2012, respectively. In 2012, he joined the Optical Communications Group, Department of Electrical and Electronic Engineering from Universidad Publica de Navarra. He has been a visiting Ph.D. student at the School of Engineering and Mathematical Sciences at City University of London. His research interests are in fiber optic lasers, interferometric sensor systems, fiber sensor networks and multiplexing architectures.

Verónica de Miguel was born in La Rioja, Spain, in May 1990. She received the telecommunication engineering degree from the Universidad Publica de Navarra, Spain in 2014. In 2015, she joined the Optical Communications Group, Department of Electrical and Electronic Engineering from Universidad Publica de Navarra. Her research interests are in fiber optic lasers, optical fiber sensor networks and multiplexing architectures.
Rosa Ana Perez-Herrera received the telecommunications engineering degree from the University of Cantabria, Spain, in 2004. In 2005 she joined the Optical Communications Group at the Department of Electrical and Electronic Engineering of the Public University of Navarra (Pamplona, Spain). During this period, she was a visiting Ph.D. student at INESC in Unidade De Optoelectronica E Sistemas Electronicos in Porto (Portugal), at the School of Engineering and Mathematical Sciences at City University of London and at the Department of Information Engineering at Parma University (Italy) among others. In 2009, she became an Assistant Professor in the Electrical and Electronic Engineering department of the Public University of Navarra. In 2010 she obtained her Ph.D. degree from the Public University of Navarra, Spain. She has coauthored more than 60 works in conferences and international refereed journals related with fiber-optic amplifiers and lasers, robust networks, and fiber-optic sensors.

Mikel Bravo Acha received the Ph.D degree from the Universidad Pública de Navarra in February 2015. The topic of his doctoral dissertation was on photonics systems for optical fiber sensors and fiber based optical sources. He was research visitor in the INESC Porto and the City University London laboratories. Nowadays, he is collaborating with the Optical Communations Group of the Universidad Pública de Navarra. He is author or co-author of over 40 papers in international refereed journals and conference contributions. His current research interests include interferometric optical fiber sensors, the random distributed feedback effect for optical sources, optical sensing and its application in real environments.

Manuel López-Amo (M'91, SM '98) was born in Madrid, Spain, in 1960.He received the M.Sc. telecommunication engineering degree and the Ph.D. degree from the Universidad Politécnica de Madrid, Spain in 1985 and 1989, respectively. From 1985 to 1996, he belonged to the Photonic Technology Department of the Universidad Politécnica de Madrid, where in 1990 he became an associate professor. In 1996, he moved to Public University of Navarra (Pamplona, Spain) where he became a Full Professor in the Electrical and Electronic Engineering department and is currently the head of the optical communications group of this department. He has been chairman of the Optoelectronic Committee of Spain. He has been leader of more than 50 research projects and he has coauthored more than 250 works in international refereed journals and conferences related with fiber-optic networks, optical amplifiers, fiber-optic sensors, and integrated optics. $\mathrm{He}$ is a member of the technical committees of the International Conference on fiber optic sensors (OFS), the European Workshop on optical fiber sensors (EWOFS), and the Asia Pacific Optical Sensors Conference, among others. Professor López-Amo is senior member of the IEEE and member of the OSA. 